\title{
Minimal function on 3-manifolds with boundary
}

\author{
N.V. Lukova-Chuiko
}

\begin{abstract}
We construct the complete topological invariant of minimal functions on the three-dimensional manifolds and proved the theorem about the implementation of this invariant feature. Thus, it is received the minimal topological classification of functions. Efficiency of constructed invariants is demonstrated by examples. We describe all the functions, the complexity of which does not exceed three.
\end{abstract}

Keywords Minimal function, topological equivalence, 3-manifolds, classification

УДК 550.837; 550.37.382

Mathematics Subject Classification (2000) 58K05

We consider functions on compact 3-manifolds with a boundary that does not have critical points such that their restrictions on the boundary have no more than 4 critical points. The main purpose of work is to give their topological classification. For Morse functions with four isolated critical points on a closed manifold, topological classification was obtained in [1,2]. The case of functions with an arbitrary number of critical points on the surface was considered in [3].

Let consider the Morse function $f$ on 3 -manifold $M$, the restrictions of which to the boundary is a Morse function. Such functions are called m-functions. Their layered classification was obtained in [4]. Connecting the internal critical point of $\mathrm{m}$-functions and pathways verge of throwing the vicinity of these roads, we get manifold, that is diffeomorphic to initial one and m-function without inner critical points on it. Index ind $x$ of critical point on the bundary is a pair $(\lambda, \delta)$, where $\lambda$ is conventional index of function restriction to the boundary (negative 
number of members in Morse theorem) and $\delta=+1$, if the vector $\operatorname{grad} f \operatorname{directed}$ outwards and $\delta=-1$ if $\operatorname{grad} f$ directed into the manifold $M$. Further, we can streamline critical points so as to first go to High point index $(0,-1)$ then $(0,1)$, $(1,-1),(1,1),(2,-1)$ and end $(2,1)$. All index point $(0, \delta)$ and $(2, \delta)$, but one of the index $(0,-1)$ and one index $(2+1)$, with points decreasing index $(1, \delta)$.

Let $M$ is a connected compact three-dimensional manifold with boundary coherent, is not homeomorphic three-disc, $f: M \rightarrow R$ is a smooth function on it. Function $f$ is called minimal function if it has no critical points and limit it to a limit of no more than four critical points that provided the growth of the function are as follows: 1$)$ the minimum point of index $(0,-1) ; 2)$ (degenerate) point of $\delta=-1 ; 3$ ) (degenerate) point of $\delta=+1 ; 4)$ the maximum point with index $(2,+1)$. Minimum function is the minimal number of critical points of functions on a three-dimensional manifold.

\section{The minimal function diagram}

Let's consider how the level surface of the minimum functions is changed with increasing the function value. When passing through the minimum point, that point of index $(0,-1)$, there is a two-dimensional disk. Further, the absence of critical points don't change the topological type of surface. In the neighborhood of a degenerate critical point, function $f$ is topologically equivalent to the functions $\operatorname{Re}(x+\mathrm{i} y)^{k}$ for some natural $k$ [3]. Then the function $f$ is topologically equivalent to functions $\operatorname{Re}(x+\mathrm{i} y)^{k}+z$ in coordinates $x, y, z, z \geq 0$. With the passage of second point, two-dimensional $2 \mathrm{k}$-gon is attached. Until the manifold joins the so-called generalized m-handle, that equivalent attaching $k-1$ usual m-handles. So, after passing the second point, we get the level surface will be some surface $F$, where is selected $k$ properly imbedded segments $u_{1}, u_{2}, \ldots$, $u_{k}$ (end pieces are on the verge of surface), without intersections and points of selfintersection. Set $u=\left\{u_{1}, u_{2}, \ldots, u_{k}\right\}$ of correctly nested curves that do not intersect each other in the surface $F$ such that after cutting them we get two disks, and each $u_{i}$ belongs to the boundary of each of these discs is called a cut system of the surface $\mathrm{F}$.

Draw a similar construction to function $-f$. We get the surface $F /$ and the cut system $v^{\prime}=\left\{v_{1}^{\prime}, v_{2}^{\prime}, \ldots, v_{k}^{\prime}\right\}$ on it. As between the levels $F$ and $F^{\prime}$ no critical points, they are homeomorphic to each other, and between them manifold homeomorphic to $F \times[0,1]$. This direct product of different structures correspond to different but isotopic homeomorphisms $F^{\prime}$ on $F$. Let $v=\left\{v_{1}, v_{2}, \ldots, v_{k}\right\}$ be the image of sections system $v^{\prime}=\left\{v_{1}^{\prime}, v_{2}^{\prime}, \ldots, v_{k}^{\prime}\right\}$ at one of these homeomorphisms. 
If the surface $F$ is oriented, we fix the orientation on it. Then the orientation of 2-disc that correspond to minimum of function, is induced orientation on its boundary, including sections on $u$. Similarly, the orientation of the disk 2 , corresponding to maximum, creates the orientation of the curves $v$. In the case of non-oriented surface we fix the arbitrary orientation of these two 2-disc, and also obtain an orientation of curves $u$ and $v$.

The triple $(F, u, v)$ is called the function diagram. Diagrams $(F, u, v)$ and $\left(F^{\prime}, u^{\prime}, v^{\prime}\right)$ are called homeomorphic if there exists a homeomorphism $h: F \rightarrow$ $F^{\prime}$, that $h(u)=u^{\prime}, h(v)=v^{\prime}$. However, if the homeomorphism changes the orientation of surface, that it changes the orientation of cuts. In the case of non-oriented surface homeomorphism preserves or simultaneously changes the orientation of u-cuts and, similarly, v-cuts.

Diagrams $(F, u, v)$ and $\left(F^{\prime}, u^{\prime}, v^{\prime}\right)$ are called semiisotopic if there id the isotopy $\varphi_{t}, \phi_{t}: F \rightarrow F$, that $\varphi_{0}=\phi_{0}=\mathrm{id}, \varphi_{1}(u)=u^{\prime}, \phi_{1}(v)=v^{\prime}$. So, different structures of direct product on $F \times[0,1]$ lead to semiisotopic diagrams. Using semiisotopic diagrams we can destroy all curved twoangles in which one party system belongs to $u$, and another $v$. You can also destroy all curved triangles in which the one party system belongs to $u$, the second to $v$, and the third to $\partial F$. Also, using semiisotopic curves of the systems $u$ and $v$, which isotope can be done such that their coincide. The diagram, which has no curved twoangles and triangles, and isotopic curves from different systems and concordant, we call normalized.

Theorem 1 Minimum functions $f: M \rightarrow R, g: N \rightarrow R$ are topologically equivalent if and only if when the normalized diagram which is built on them is homeomorphic.

Proof. Necessity. During construction, the diagram of the function is unique up to semiisotopy. Similarly proof of Theorem 5.3. in [2], if Heegaard diagrams are semiisotopic, then the normalized diagrams are homeomorphic. Furthermore, the topological equivalence of functions generates the homeomorphism between diagrams and, consequently, the homeomorphism between normalized diagrams.

Sufficiency. If the diagrams are homeomorphic, then they have the same number $k$. Then, in the neighborhoods of the critical points, the functions are topologically equivalent to function $h(x, y, z)=\operatorname{Re}(x+i y)^{k} \pm z$ or $h(x, y, z)=$ $\pm\left(x^{2}+y^{2}+z\right)$. So, the functions are topologically equivalent in the neighborhoods of the critical points. As for Heegaard diagrams [2], homeomorphism of normalized diagrams sets the extension of homeomorphisms to the desired homeomorphism of three-dimensional manifold. 


\section{Graph of the function}

We construct an oriented graph which is embedded in a closed surface and called by the graph of the function. We contract each component of the boundaries to a point which will be considered as vertices. We consider the cuts $u_{1}, u_{2}, \ldots, u_{k}$ and $v_{1}, v_{2}, \ldots, v_{k}$ as the edges of the graph. If the cuts coincide, they will meet one "double" edge. Thus, the edges of the graph are divided into three types: 1) $u$;2) $v$; 3) double. The orientation of cuts sets the orientation of edges. Thus, the double edges have two orientations ( $u$-orientation and $v$-orientation). After contracting of the boundary component of $F$ at the point, we obtain a new closed surface $\Phi$. Thus, the graph of the function is embedded in $F$. We will consider such graphs in which more than two edges (cases with 1 and 2 edges we will discuss in the examples).

Theorem 1 Minimal functions $f: M \rightarrow R, g: N \rightarrow R$ are topologically equivalent if and only if there exists a homeomorphism of corresponding surfaces $F$, which sets isomorphism of the graphs, preserves the type of the edges and preserves or simultaneously changes the orientation of edges as for cuts.

Proof. Necessity. Necessity is followed from Theorem 1 and unambiguous constructing a graph of functions.

Sufficiency. Let show how to build a diagram by the graph of function. Cut from the surface $S$ regular neighborhood of vertices. We obtain the surface which is homemorphic to $F$ and imbedded curves (sections) that are set be embedding of edges of the graph. A couple of double edges corresponds to $u$ - and $v$-cuts that match. Then the homeomorphism of surfaces $F$ sets homeomorphism of graphs, and, consequently, the topological equivalence of functions. The theorem is proved.

Properties of the graph of the function. By constructing a graph of function, it has the following properties:

1) the number of edges of type $u$ is equal to the number of edges of type $v$;

2) $u$-subgraph that consist of edges of type $u$ and double edges divides the surface into two 1-connected region and each edge of this part of the subgraph belongs to the boundary of each of the two regions;

3) similar properties for $v$-subgraph made up of v-type edges and double edges.

Theorem 2 A graph which is embedded in a closed surface, the edges of which are divided into three types, so running properties 1) - 3), is the graph of some minimal function. 
Proof. Let consider four 3-dimensional disks and functions $h(x, y, z)=\operatorname{Re}(x+$ $i y) k \pm z$ and $h(x, y, z)= \pm\left(x^{2}+y^{2}+z\right)$ on them, where $(0,0,0)$ - some point on the boundary in the neighborhood of which disk is given by $z \geq 0$. The graphs of the functions construct a diagrams which sets gluing these disks together. After gluing we obtain three-dimensional manifold and function on it. If it necessary, smoothing function in places of gluing we obtain the needed function.

\section{Examples and calculations}

The number of cuts $k$ for one of the systems will be called the complexity of the function. If $F$ is an oriented surface of genus $g$ with $d$ components of the boundary, then $k=2 g+d$, and if $F$ is non-oriented surface, then $k=g+d$.

Let consider the case of oriented surface.

1) $k=1$. Then $g=0, d=1$. There is only one graph of the function double loop edge. Two orientations on it it may be the same or be opposed. But both these cases set semiisotopic diagrams. Thus, there exists a unique, up to topological equivalence, minimal function.
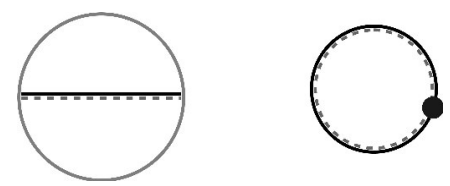

Fig. 1.

2) $k=2$. Then $g=0, d=2$. There is also a single graph, which is homeomorphic to circle with two vertices on it and double edges, and both pairs of orientations for it lead to semiisotopic diagrams. Thus, there exists a unique, up to topological equivalence minimal function in this case.

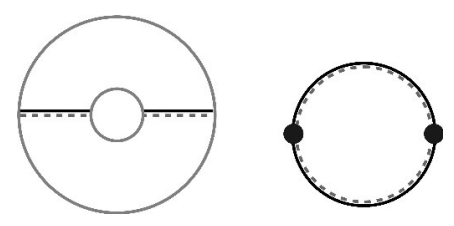

Fig. 2.

3) $k=3$. A) $g=0, d=3$. The graph is homeomorphic to circle with three vertexes on it and double edges. In the double edges it may be the same or opposite orientation. So there are two, up to topological equivalence, minimal function. 


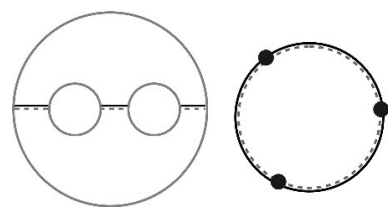

Fig. 3.

B) $g=1, d=1$. $u$-subgraph has one vertex and three loop without intersections on the torus. Two of these loops are generating of fundamental group of the torus, and the third is their product. $v$-subgraph has the same property. Since pair of generators of the fundamental group is expressed through another pair of generators via a matrix of $\operatorname{SL}(2, Z)$, then the matrix sets the graph of the function. These two matrices set the same graph of the function, if they are the same, or one of the other available matrix multiplication to -1 . Consequently, there are infinitely many graphs in this case and therefore minimal functions. Fig. 4 shows a $u$-subgraph and graph of the matrix $\left(\begin{array}{ll}1 & 0 \\ 2 & 1\end{array}\right)$.

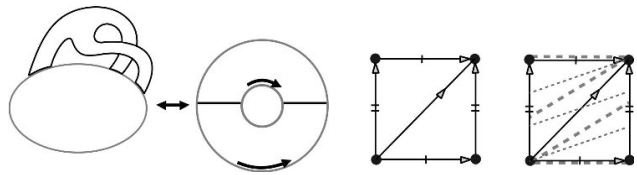

Fig. 4.

Let consider the case of non-oriented surface.

1) $k=2$. Then $g=1, d=1$. There is only one graph which is homeomorphic to 8 with double edges. $F$ is homeomorphic to Mobius strip. If it is represented as a rectangle with a collapsible pair of opposite sides, then it cuts homotopic to sides. The same and opposite orientation of double edges set semiisotopic diagrams. Thus, there exists a unique, up to topological equivalence, minimal function.
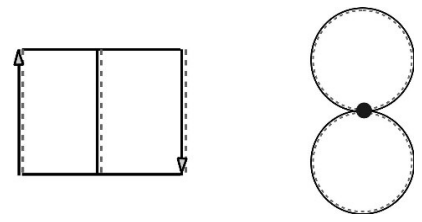

Fig. 5.

2) $k=3$. A) $g=1, d=2$. $u$-subgraph can be obtained from the graph of the functions as in the previous example, if you add one of the top on one of the edges, thus breaking it in two edges. A graph can be obtained if all edges attribute type double edges. Another graph looks like two 8 with shared circle. 
Each of the double edges, each pair of orientations can be selected in two ways. Then there are all four methods for the couple. Thus, there are eight, up to topological equivalence minimal functions.

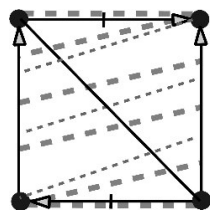

Fig. 6.

B) $g=2, d=1$. $u$-subgraph has one vertex and three loops. As in the case of oriented $3 \mathrm{~B}$ ), the torus with a hole get infinitely many graphs. For example, Klein bottle is represented as a square, which cemented opposite directions. One pair of vertices submits preserving orientation, and the rest of the rotation submits the matrix $\left(\begin{array}{ll}1 & 0 \\ n & 1\end{array}\right)$ as the torus set the graphs. The example when $\mathrm{n}=$ 3 is shown in Fig. 7.
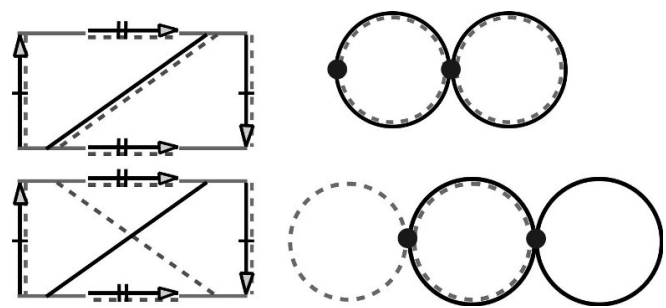

Fig. 7.

Thus, all the functions the complexity of which does not exceed three are described.

\section{References}

1. A.O. Prishlyak, The topological sructure of the functions with isolated critical points on 3-manifold // Algebras, Groups and Geometries, Vol. 32, No. 4, 2015.- pp. 400-422.

2. A. Prishlyak. Topological properties and functions on 2- and 3-manifolds. Palmarium Academic Publishing 2012. 132s.

3. V.M. Kuzakon, V.F.Kirichenko, A.O.Prishlyak. Smooth manifolds. Geometric and topological aspects // Proceedings of Inst Mathematics NAS of Ukraine. Mathematics and its applications. - 2013. - V. 97. 500 p.

4. N.V. Lukova-Chuiko, A.O. Prishlyak. M-layered equivalence of functions in general position on 3-manifolds with boundary // Journal of Computational and Applied Math. - 2011.- No.3 (106) - c.114-123.

\section{N.V. Lukova-Chuiko}

Taras Shevcheno National University of Kyiv

E-mail: lukova@ukr.net 